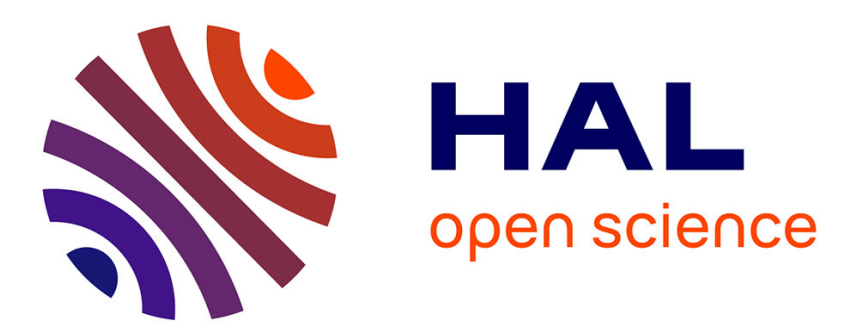

\title{
Les Juifs du bassin de Lens face à leurs voisins : concurrences, dénonciations, entraides (1940-1945) \\ Nicolas Mariot, Claire Zalc
}

\section{To cite this version:}

Nicolas Mariot, Claire Zalc. Les Juifs du bassin de Lens face à leurs voisins : concurrences, dénonciations, entraides (1940-1945). Didier Terrier \& Judith Rainhorn (dir.). Étranges voisins. Altérité et relations de proximité dans la ville depuis le XVIIIe siècle, Presses universitaires de Rennes, pp.237253, 2010, 9782753510111. 10.4000/books.pur.127740 . hal-03417784

\section{HAL Id: hal-03417784 \\ https://hal.science/hal-03417784}

Submitted on 5 Nov 2021

HAL is a multi-disciplinary open access archive for the deposit and dissemination of scientific research documents, whether they are published or not. The documents may come from teaching and research institutions in France or abroad, or from public or private research centers.
L'archive ouverte pluridisciplinaire HAL, est destinée au dépôt et à la diffusion de documents scientifiques de niveau recherche, publiés ou non, émanant des établissements d'enseignement et de recherche français ou étrangers, des laboratoires publics ou privés. 


\title{
Les Juifs du bassin de Lens face à leurs voisins : concurrences, dénonciations, entraides (1940-1945)
}

\author{
Nicolas Mariot et Claire ZaLC
}

L'histoire de la Shoah a fait l'objet, depuis une quinzaine d'années, d'un grand nombre de travaux, le plus souvent menés dans le cadre d'une histoire des persécutions, de leur chronologie et de leurs effets ${ }^{1}$. Les politiques de discrimination antisémite ont d'abord été analysées " par le haut ", du point de vue du rôle de l'État et des acteurs institutionnels qui y ont participé ${ }^{2}$. Dans une perspective complémentaire, nous voudrions ici suggérer que ces processus peuvent être perçus et pensés à partir des liens de voisinage.

Les historiens américains Eric Johnson et Robert Gellately ont montré, pour l'Allemagne nazie, que l'encadrement de la société par la Gestapo n'aurait pu être réalisé par les seuls effectifs, limités, du corps ${ }^{3}$. Ainsi, le travail de la police politique allemande sous le Troisième Reich a consisté, pour une large part, à traiter les dénonciations qui lui parvenaient. D'autres auteurs, sur le cas français, commencent à analyser les processus de "repérage " des individus mais également des biens " juifs " via l'étude du phénomène de dénonciation pendant la Seconde Guerre mondiale ou encore celle des processus d'aryanisation des biens ${ }^{4}$. Dans cette optique,

\footnotetext{
1. Pour des perspectives européennes générales, voir Raul HiLberg, La Destruction des Juifs d'Europe, Paris, Gallimard, coll. "Folio Histoire ", 2006, édition définitive, complétée et mise à jour (édition originale : 1961, en anglais), 3 volumes; Florent BRAYARD, La "solution finale de la question juive ". La technique, le temps et les catégories de la décision, Paris, Fayard, 2004 et Saul FrIEDLÄNDER, Les années d'extermination. L'Allemagne nazie et les Juifs, 1939-1945, Paris, Seuil, 2008. Pour la seule zone occupée en France, la synthèse récente de Thomas Fontarne, "Répression et persécution en France occupée. 1940-1944 ", Online Encyclopedia of Mass Violence, http://www.massviolence.org/
PdfVersion?id_article=84\&lang=fr.

2. Michaël R. MARRUS

Peschanski, Vichy, 1940- Roert O. Paxton, Vichy et les Juifs, Paris, Calmann-Lévy, 1981; Denis Au Bureau des Affaires Juives. Contrôle et exclusion, Bruxelles, Complexe, 1997; Tal Bruttmann, (1940-1944), Paris, La Découverte, 2006.
} 3. Robert Gellately, The Gestapo and German Society: Enforcing Racial Policy, 1933-1945, Oxford, 1991
et "Denunciations in Twentieth-Century Germany: Aspects of Self-Policing in the Third Reich and the
German Democratic Republic", Journal of Modern History, German Democratic Republic ", Journal of Modern History, 68, 1996, p. 931-967; Eric A. JoHNson, La terreur nazie. La Gestapo, les Juifs et les Allemands ordinaires, Paris, Albin Michel, 2001 [éd. orig. 1999]. délation antisémite sous ", Revue d'histoire de la Shoah, n ${ }^{\circ} 186$, janvier-juin 2007, Laurent Joly, "La délation antisémite sous l'Occupation ", Vingtième Siècle. Revue d'histoire, n 96, 2007, p. 137-149. 
nous souhaitons montrer ici que les opérations de discrimination et d'aryanisation menées à l'encontre des Juifs de Lens (Pas-de-Calais) entre 1940 et 1945 ne peuvent être comprises si elles ne sont pas encastrées dans les liens sociaux de voisinage, faits d'entraides, de dénonciations, mais aussi d'indifférence. Cette démarche permet de revenir sur la notion de voisinage, comprise comme une relation de proximité spatiale ou temporelle, mais également pensée comme une relation de proximité professionnelle, économique ou encore sociale. Nous aimerions ainsi montrer que le " voisinage " ne peut être appréhendé dans une seule acception géographique, réduite à une mesure des liens spatiaux. Il est nécessaire de comprendre la notion en pratique, incarnée dans des relations interpersonnelles aux conséquences parfois dramatiques en contexte de violence extrême.

En second lieu, la perspective monographique permet de contextualiser au mieux les observations puisqu'elle amène à saisir les effets de ces politiques " au ras du sol ", quartier par quartier, rue par rue, immeuble par immeuble ${ }^{5}$. Le cas lensois est, en ce sens, particulièrement intéressant parce qu' on dispose d'un riche matériel archivistique ${ }^{6}$. Trois types de sources ont été mobilisés : les archives émanant des institutions policière et administrative locales, conservées aux Archives départementales du Pas-de-Calais (Arras); les documents propres à la politique d'aryanisation économique menée par le Commissariat général aux questions juives, dossiers conservés dans la série AJ 38 des Archives nationales; enfin les données recueillies par le Centre de documentation juive contemporaine, notamment les listes des convois de déportation, permettant de disposer des traces des personnes arrêtées et déportées de Lens ou d'autres localités en France.

Notre propos, centré sur les procédures d'aryanisation, sera structuré en plusieurs temps : après avoir dessiné les contours de l'inscription locale des Juifs dans le bassin lensois pendant la Seconde Guerre mondiale, on suivra comment, à chaque étape de la procédure discriminatoire, les voisins, ou plus exactement, le voisinage, a, ou non, joué un rôle. L'enjeu est double : d'une part, tenter sur ce cas spécifique de qualifier la relation de voisinage dans un contexte extrême en comprenant quels types de liens elle recouvre, la proximité spatiale n'étant pas nécessairement, par elle-même, génératrice de relations sociales; d'autre part, fournir, par l'évocation du concret de la politique de discrimination antisémite dans le bassin lensois, des éléments

5. Dans une perspective comparative, voir par exemple Renée Dray-Bensousan, Les Juifs à Marseille durant la Seconde Guerre mondiale, 1940-1944, Paris, Les Belles Lettres, 2004; Jean EstèBE, Les Juifs à Toulouse et en Midi-Toulousain au temps de Vichy, Toulouse, Presses universitaires du Mirail, coll. "Tempus ", 1996.

6. Ce travail est né dans le prolongement d'une enquête collective ancrée sur la région lensoise, exposée notamment dans Françoise DE BARROS et Claire ZALC, "Faire parler des archives, historiciser un terrain : les salariés d'une entreprise familiale (Lens, 1945-1975) "Anne-Marie Arborio et alii, (dir.), Observer le travail. Histoire, ethnographie, approches combinées, Paris, La Découverte, coll.« Recherches ", 2008, p. 45-60. Nous assumons néanmoins ici toute la responsabilité des propos tenus. 

pour réfléchir à l'insertion locale de cette politique en France pendant la
Seconde Guerre mondiale.

\section{Une implantation urbaine resserrée}

La " communauté juive " du bassin lensois, au contraire des communautés parisienne ou lilloise, est une communauté créée ex nibilo durant l'entre-deux-guerres, composée pour plus de $80 \%$ d'immigrants d'Europe centrale et orientale arrivés en France dans les années 1920 et 1930. Ils s'installent pour l'essentiel dans la ville de Lens : sur 926 individus identifiés comme " juifs " pendant la Seconde Guerre mondiale dans le bassin lensois (comprenant l'ensemble des communes du bassin houiller), plus de $80 \%$ sont domiciliés à Lens $(772)^{7}$. Pour la majorité d'entre eux, ils se spécialisent dans la vente ambulante de vêtements et de tissus aux immigrants polonais installés au début des années 1920 dans les corons ${ }^{8}$.

Pour autant, s'ils entretiennent avec les mineurs polonais des relations linguistiques et commerciales, ils ne sont que très rarement voisins de cette population, au sens géographique du terme. En effet, les mineurs logent à proximité des puits, dans les nombreuses « cités » qui composent la périphérie du territoire de la commune lensoise. Au contraire, la grande majorité des Juifs lensois identifiés en 1940 logent dans un rayon de 1300 mètres autour de la gare et, à vrai dire pour $95 \%$ d'entre eux, dans le demi-cercle constituant le centre-ville commerçant au nord de la voie ferrée (carte 1). Il semble ainsi possible d'affirmer qu'ils vivent à quelques minutes (ou quelques centaines de mètres) les uns des autres. Peut-on alors parler de " ghetto " juif à Lens à la fin des années 1930?

La carte 1 fait état de l'implantation des familles, en distinguant les rues comptant 1 à 4 ménages identifiés comme juifs (gris clair), celles où l'on en dénombre de 5 à 9 (gris foncé), enfin celles où vivent plus de 10 familles (noir). Le figuré utilisé (coloration des rues) peut donner l'impression d'un petit ghetto, mais il n'en est rien : si l'on pondère le nombre de ménages juifs par le nombre de familles habitant dans les rues, grâce à l'analyse des listes nominatives des recensements de l'entre-deux-guerres, on constate que seules deux d'entre elles (les rues F. Gauthier et Decombecque) comptent $30 \%$ de ménages identifiés comme juifs, l'ensemble des autres ne dépassant

\footnotetext{
7. Sur la constitution et les frontières sociales et géographiques de la " communauté israélite de Lens 1936), voir notre retient ici la définition indigène du groupe lors de sa déclaration en préfecture en 1944) ", dans Gérard Norrier (dir.), L'iden problèmes de l'identification des Juifs lensois (1940"Socio-histoires ", 2007, p. 181-200. Lidentification. Genèse d'un travail d'État, Paris, Belin, coll.

8. Martina Avanza, Gilles LAFerTÉ, Ni

entreprise entre deux "communautés" à LARIOT et Claire ZaLC, "Habiller les mineurs : une (dir.), Aux marges de la mine. Représentations, stratérgess 1945 ", Jean-François ECK et Didier TERRIER de-Calais, XVIIT'-XX' siècles, Valenciennes, Presses valenciennoises " $n^{\circ} 23,2007$, p. $165-190$.
} 
pas $20 \%$. On retrouve ici le constat déjà établi pour la région parisienne dans l'entre-deux-guerres : l'observation large conduit à se focaliser sur les phénomènes de concentration. Mais lorsque le regard se fait plus fin, il met au jour la pluralité des logiques d'implantation des immigrants et la mixité des quartiers?

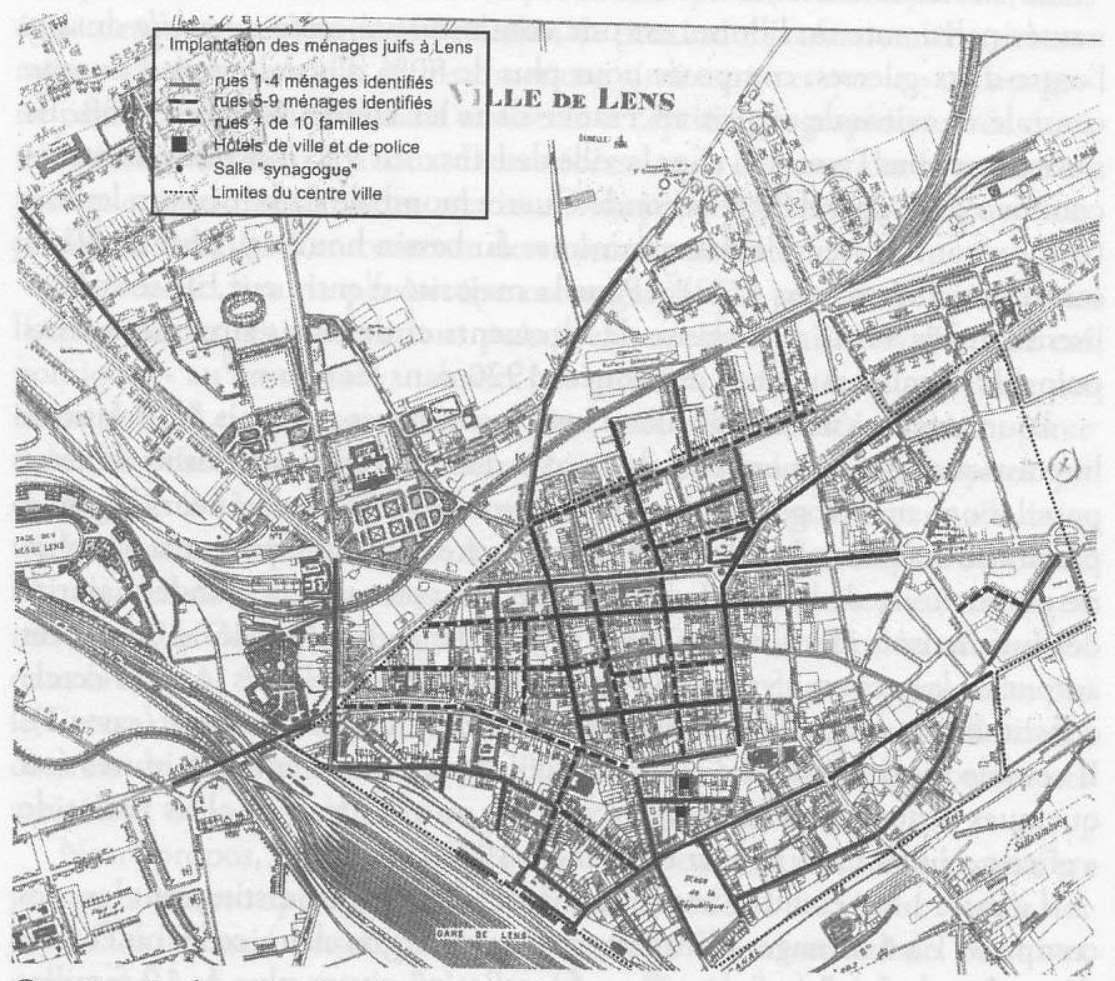

Carte 1. - Implantation des ménages juifs à Lens.

Ce constat est d'importance au sens où il permet de prendre conscience du relatif isolement de certaines familles. Cet isolement protège et stigmatise en même temps, lors des processus d'identification de ces ménages comme "juifs ", processus mis en œuvre par les autorités préfectorales, municipales et policières du Pas-de-Calais suite aux ordonnances édictées dès l'automne 1940 par les autorités allemandes d'occupation de Bruxelles dont dépend la "zone interdite ${ }^{10}$ ". Mais la relation aux autres, et plus

9. Marie-Claude BlANC-ChALÉARD, "L'habitat immigré à Paris aux $\mathrm{XIX}^{\mathrm{e}}$ et $\mathrm{XX}^{\mathrm{e}}$ siècles : mondes à part? ", Le Mouvement social, $\mathrm{n}^{\circ}$ 182, janvier-mars 1998, p. 29-53; Claire ZALC, "Petits entrepreneurs étrangers en ville. Implantations urbaines, réseaux migratoires et solidarités professionnelles à Paris dans l'entre-deux-guerres ", Histoire urbaine, n 3, décembre 2001, p. 67-82.

10. Nicolas Mariot et Claire ZALC, "Identifier, s'identifier : recensement, auto-déclarations et persécution des Juifs lensois (1940-1945) ", Revue d'histoire moderne et contemporaine, vol. 54, $\mathrm{n}^{\circ} 3$, juillet-sept. 2007, p. 91-117. 

spécifiquement aux voisins, se rejoue à chaque étape de la discrimination
des Juifs lensois entre 1940 et 1945 .

\section{L'aryanisation, une affaire de voisins?}

C'est l'ordonnance du 18 novembre 1940, relayée par l'Oberfeldkommandantur 670 de Lille, qui institue un recensement des Juifs et prévoit, dans le Nord-Pas-de-Calais, la nomination d'un administrateur provisoire pour toute entreprise industrielle, commerciale, immobilière ou artisanale, ainsi que pour tout immeuble ou bien meuble "lorsque ceux à qui ils appartiennent ou qui les dirigent ou certains d'entre eux sont juifs ». Dans une lettre au préfet du Pas-de-Calais en date du 9 décembre 1940 , le Militärbefehlshaber de Bruxelles donne l'ordre que toutes les entreprises " juives " et magasins " juifs " ouverts sur rue soient pourvus d'un commissaire gérant. Le 15 décembre 1940, il est précisé : "Ces mesures d'aryanisation ne sauraient avoir pour objet que d'éliminer l'influence israélite de l'économie nationale, étant entendu que les intérêts actuellement détenus par les israélites qui tombent sous le coup des ordonnances allemandes devront, de manière générale, demeurer entre des mains françaises ${ }^{11}$. »

Nous ne reviendrons pas ici sur le déroulé et les modalités du processus de spoliation des biens des juifs lensois, sur lesquels nous avons pu dépouiller une abondante documentation et qui s'inscrit dans un mouvement général, décrit pour de nombreuses régions depuis les travaux de la mission Matteoli ${ }^{12}$. Nous aimerions plutôt décrire l'aryanisation des biens " juifs » au prisme d'une analyse des relations de proximité. En effet, le processus de spoliation peut et doit sans doute être lu de manière spécifique dans les petites communautés locales de province : loin de s'apparenter au résultat du travail anonyme et administratif des relais de l'État central, l'aryanisation s'insère dans le réseau économique au sein duquel les entrepreneurs exerçaient jusque-là leur activité.

Tout d'abord, rappelons que les différents acteurs du processus d'aryanisation, commissaires gérants et administrateurs provisoires, chargés de liquider ou d'organiser le transfert de propriété des biens dits " juifs », sont très souvent des " voisins » des entrepreneurs et/ou propriétaires spoliés. Les administrateurs provisoires sont nommés par les autorités allemandes (le Militärbefehlshaber de Bruxelles) puis désignés par un arrêté ministériel du

11. Archives départementales du Pas-de-Calais (désormais ADPC), $1 Z 498$.

12. Alya AgLan, «L'Laryanisation des biens juifs sous Vichy : les cas comparés gne ", Revue d'histoire moderne et contemporaine, vol. $49, \mathrm{n}^{\circ} 4$, octobre-décem la France et de l'AllemaLaurent Douzou et al., Voler les Juifs, Lyon, 1940-1944, Paris, Hachette, 2002; Jean-Marc DreYFus,
Pillages sur ordonnances : aryanisation et restitution des banques en 2003; Florent Le Bot, «Dans les filets des spolion des banques en France, 1940-1953, Paris, Fayard, parisiennes sous l'Occupation ", Archives juives de Frans : les échoppes et les petites fabriques de cuir Philippe Verheyde, Les mauvais comptes de Vichy : l'aryanisation ${ }^{\circ}$, $2^{\circ}$ semestre 2006, p. 42-63; 1999. 
Commissariat général aux questions juives (CGQJ) publié au Journal officiel. Le choix du commissaire gérant relève du préfet du Pas-de-Calais qui doit se conformer aux instructions suivantes, énoncées par le Militärbefehlshaber le 15 décembre 1940 :

" J'insiste sur ce fait que, dans tous les cas, votre choix ne saurait porter que sur des hommes à la fois compétents dans la branche professionnelle considérée, dégagés de toute influence des anciens dirigeants et animés de manière incontestable de la volonté de contribuer au relèvement de l'économie nationale, à l'exclusion de toute considération de personne ou d'intérêts particuliers ${ }^{13}$."

À Lyon, il s'agit de candidatures spontanées, envoyées par lettres, et essentiellement à partir de juillet 1941. Puis la préfecture intervient pour examiner les candidatures, et leur attribuer des biens à gérer ${ }^{14}$. Nous n'avons pas trace, dans les archives concernant le bassin lensois, de volontaires se portant candidats à l'aryanisation. Par contre, la proximité spatiale joue un rôle dans l'attribution des biens à gérer.

S'il est prévu que, pour les entreprises importantes, un commissaire gérant ne soit pas chargé de plus de cinq entités, pour les petites entreprises, qui composent la grande majorité de l'ensemble ici, les modalités de nomination des commissaires gérants prennent, dès les instructions initiales, une dimension territoriale très forte puisqu'il est prévu de nommer " par commune ou quartier de ville, un commissaire collectif pour toutes les entreprises d'une branche professionnelle déterminée ${ }^{15}$ ". Néanmoins, le nombre d'entreprises attribuées à chaque commissaire gérant dans le bassin lensois varie grandement. La moyenne se situe à un peu moins de quatre entreprises par commissaire gérant (pour 110 entreprises ou immeubles, on compte 29 commissaires gérants différents), mais la dispersion est importante : parmi eux, cinq sont chargés de gérer plus de dix entreprises, alors que vingt n'en ont qu'une seule.

Surtout, la localisation géographique joue un rôle important dans les processus de nomination, comme en témoigne la mise en relation des adresses des biens spoliés avec celles des commissaires gérants dans un tableau à la diagonale éloquente (tableau 1).

Pour venir conforter la lecture en termes de voisinage, il faut préciser que dans la grande majorité des cas, les adresses des entreprises et/ ou bien spoliés sont les adresses des commerçants et/ou propriétaires. On peut mettre en lumière grâce à une comparaison systématique des adresses, la constitution d' " aires d'intervention " des commissaires gérants lensois situées relativement souvent dans un voisinage proche de leur domicile.

13. ADPC, 12499 .

14. Laurent Douzou, Voler les Juifs, op. cit., p. 108 et suivantes.

15. Lettre du préfet du Pas-de-Calais du 15 décembre 1940, ADPC 1 Z499. 


\begin{tabular}{|c|c|c|c|c|c|c|c|c|c|c|}
\hline \multicolumn{11}{|c|}{ Commune des biens spoliés } \\
\hline $\begin{array}{l}\text { Domicile des } \\
\text { commissaires } \\
\text { gérants }\end{array}$ & 通 & 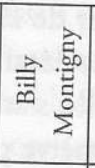 & 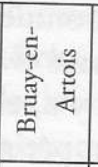 & 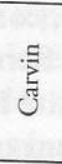 & 胥 & 声总 & $\tilde{\breve{G}}$ & 紊 & 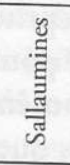 & స్ّ \\
\hline Arras & 3 & & & & & & & & & 3 \\
\hline Billy Montigny & & 1 & & & & & & & & 1 \\
\hline Bruay en Artois & & & 1 & & & & & & & 1 \\
\hline Carvin & & & & 2 & & & & & & 2 \\
\hline \begin{tabular}{|l|}
$\begin{array}{l}\text { Fouquières Les } \\
\text { Lens }\end{array}$ \\
\end{tabular} & & & & & & & 16 & & & 16 \\
\hline Harnes & & & & & 1 & & & & & 1 \\
\hline Hénin Liétard & & & & & & 2 & & & & 2 \\
\hline Lens & & & & & & & 53 & & & 53 \\
\hline Liévin & & & & & & & & 2 & & 2 \\
\hline Roubaix & & & & & & & 1 & - & 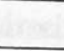 & 1 \\
\hline Sallaumines & & & & & & & & & 2 & 2 \\
\hline Inconnu & & & & & & & 5 & & & 5 \\
\hline Total & 3 & 1 & 1 & 2 & 1 & 2 & 75 & 2 & 2 & 89 \\
\hline
\end{tabular}

Tableau 1. - Des commissaires gérants situés dans les communes des biens spoliés Source: Archives nationales, AJ38 4915 à 4934 et 5951.

Mais il convient de détailler le constat, quelque peu général et globalisant, d'une "proximité spatiale " entre les commissaires gérants et les juifs spoliés. Certains exemples permettent d'ailleurs de nuancer l'existence du facteur " proximité géographique ", comme celui du principal commissaire gérant de Lens, Gambiez, en charge de 29 entreprises entre 1940 et 1944, et qui habite... une commune voisine (Fouquières-les-Lens). Huissier de justice et clerc de notaire, Gambiez s'institue comme l'un des "spécialistes » de l'aryanisation, disposant d'un capital professionnel qui joue bien plus que son capital local. Il est de loin celui auquel revient le plus de biens et fait également figure de recours lorsque le premier commissaire gérant manque à sa mission. Toutefois, si son domicile est situé à l'extérieur de la commune, l'étude dont il dépend est, quant à elle, sise place de la République, à Lens, ce qui témoigne d'une véritable insertion locale.

Cet exemple montre la nécessité de relire les liens entre " commissaire gérant ", "individu qui fait l'objet d'une procédure de spoliation " et " administration" sans en chercher un unique déterminant. La proximité entre les commissaires et les biens " juifs " dont ils ont la charge se joue sous d'autres modes que celui du simple lien spatial. Elle est d'abord d'ordre économique et se décline alors sous le signe de la concurrence. 
Tel commissaire gérant, représentant en tissus, se voit confier en priorité les entreprises de confection. Tel autre, cordonnier de Billy-Montigny, est nommé pour aryaniser le commerce de Bernard G., détaillant en chaussures dans la même commune ${ }^{16}$. Si proximité il y a, elle s'articule sur d'autres registres que le simple fait d'habiter dans un périmètre proche.

L'argument de la concurrence est d'ailleurs porté par les commissaires gérants eux-mêmes, soucieux dans leur administration de la spoliation, de "protéger " les concurrents " aryens". On retrouve ainsi sous leur plume l'argument, largement déployé dans les années 1930, de la « concurrence déloyale " des étrangers, qui justifie souvent la décision de liquider l'affaire sans procéder à une vente, afin de protéger les concurrents. À propos de l'affaire de Laja M., marchand de chaussures à Harnes, le commissaire gérant note ainsi : "L'affaire ne paraît pas vendable, l'immeuble est en location et sert actuellement d'habitation privée à la personne. Aucun matériel. Commerce où il y a pléthore ${ }^{17}$. Le voisin immédiat étant lui-même marchand de chaussures ${ }^{18}$. " En outre, au cours de la procédure d'aryanisation, interviennent souvent d'anciens confrères (concurrents ou alliés) ainsi que des employés des entrepreneurs victimes.

\section{Une spoliation " entre soi »}

Le sort réservé aux biens « juifs » est de deux ordres : la vente ou la liquidation. Cette dernière possibilité est la plus fréquente, encouragée lorsque les affaires sont de petite importance. Elle concerne plus des trois quarts des entreprises dont on connaît le sort ici (57 sur 75). Néanmoins, dans $20 \%$ des cas environ, le commissaire gérant décide de vendre. Ce sont alors les voisins directs qui entrent en jeu.

Quand l'entreprise de meubles sise 18 rue de Lille à Lens est mise en vente, une marchande installée au 52 de la même rue adresse une lettre au CGQJ où elle relate :

"Il y a environ un an deux commissaires gérants aux affaires juives venaient me demander si j'étais acheteuse d'un stock de meubles déposé dans les magasins de "Lens Mobiliers" sis rue de Lille n 18 à Lens. Je me rendis à cette adresse avec ces Messieurs; Je leur fixais mon prix qui fut accepté par ces derniers. Il y a six mois un inventaire était dressé par Maître Duruy, huissier qui accepta la mise à prix et m'assura que sitôt les pièces nécessaires signées et timbrées par les services de la Préfecture et de la Kommandantur, je pourrais disposer de ces meubles ${ }^{19}$."

16. Archives nationales (désormais AN), AJ38/6460.

17. Souligné dans le texte, AN, AJ38/4923.

18. AN, AJ38/4292.

19. AN, AJ38/4975, lettre du 12 août 1942. 
Elle dit ainsi avoir été directement sollicitée par les commissaires gérants. Mais on observe aussi à plusieurs reprises, dans les archives, la trace de voisins qui se déclarent d'eux-mêmes " intéressés " par tel ou tel bien " juif ».

C'est notamment le cas lors des mises en vente. Les modalités sont fixées par des textes : le commissaire gérant fait passer un avis dans un journal local, le plus souvent l'Écho du Nord, et recueille les offres et candidatures des acquéreurs potentiels, tenus, alors, de faire état de leur "indépendance » vis-à-vis des personnes spoliées. Les acquéreurs potentiels déclarent alors, par écrit, qu'ils n'ont aucun lien avec l'entrepreneur ou le propriétaire : "M. Gaston T. déclare sous la foi du serment qu'il est en complète indépendance vis-à-vis du propriétaire actuel et que ce propriétaire n'exercera plus désormais aucune influence sur l'entreprise, de même qu'il ne participera plus au bénéfice ${ }^{20}$. " L'analyse des listes des candidats montre cependant la présence, quasi systématique, d'un voisin qui, par sa proximité géographique, est mis au courant des vacances d'appartements ou d'entreprises ${ }^{21}$. L'appât du gain, suscité par la perspective d'une acquisition, se fait parfois au prix de reconversions professionnelles. Ainsi de ce pâtissier-confiseur spécialisé dans les baptêmes, 49 rue de Lille, qui se porte acquéreur d'une affaire de confection, robes et manteaux, située à quelques numéros dans la même rue (au 5 rue de Lille). Il remporte l'affaire, sans doute suite à un arrangement avec le commissaire gérant, qui affirme au CGQJ qu'il n'y a pas assez d'offres pour pouvoir procéder à une vente par adjudication alors que le dossier fait état d'au moins cinq offreurs déclarés à lui, suite à l'annonce parue dans l'Écho du Nord.

D'autres n'attendent pas la mise en vente pour se déclarer intéressés, dans un réflexe de prédation qui manifeste une relation de voisinage bien spécifique. La proximité spatiale favorise ce type de comportement car elle informe sur le fait qu'un bien est potentiellement "à saisir ", notamment quand la relation de voisinage se couple d'un lien économique comme ici d'une relation locataire/propriétaire : $M^{\text {me }}$ veuve Éloi C., locataire du 25 rue de la Porte d'Arras, déclare ainsi dans une lettre qu'elle " a bien l'intention de [se] mettre sur les rangs des adjudicataires éventuels " de l'affaire de Jankiel B., sise 25 porte d'Arras. Elle l'écrit le 30 mai 1943 :

"M. le préfet. J'ai l'honneur de vous prier de vouloir bien me faire connaître s'il est exact que les immeubles appartenant aux juifs vont être vendus incessamment, sous pli cacheté aux plus offrants, et dans l'affirmative les formalités à remplir pour prendre part à l'adjudication. J'exploite

20. AN, AJ38/4975.

21. Lors de la vente de l'affaire de Moïse D. à Sallaumines, offre de $\mathrm{M}^{\mathrm{me}}$ Clément, rue Séverine à Sallaumines (AN, AJ38/4932). Concernant la vente de l'entreprise de Maurice G., 37 rue de la Paix à Lens, Madame Gruson, 28 rue de la Paix à Lens se porte acquéreuse (AN, AJ38/4933). Lors d'André Bilot, ébéniste sculpteur, 45 rue Raoul Briquet à Leubles rue de la Paix à Lens, proposition 1944 car son offre est la plus élevée (AN, AJ38/4932). 
un fond de débit de boissons depuis 18 ans, à Lens, 25 rue de la Porte d'Arras dans un immeuble appartenant à un juif depuis 1940 et serais très désireuse de connaître le mode d'adjudication de ces immeubles pour tenter d'acquérir ma maison selon mes disponibilités; je suis veuve, française et
de race aryenne. En espérant ${ }^{22} \ldots »$

Les relations de voisinage se jouent également dans le cadre du lien propriétaire/locataire. Devant la disparition de leurs locataires « juifs » suite, notamment, aux rafles de septembre 1942 qui déciment la communauté lensoise, plusieurs propriétaires expriment leur intention d'occuper l'espace vacant. Monsieur Émile C., propriétaire de Billy-Montigny dépose, le 6 février 1943, une requête auprès du CGQJ : "Je possède une maison, louée sans bail à un Juif, et actuellement inoccupée. Puis-je disposer de cette maison après avoir fait procéder à l'inventaire des biens qui y restent et avoir mis ceux en garde de façon à les restituer ultérieurement au propriétaire ${ }^{23}$ ? »

Les comportements de "prédation » dérivent parfois en dénonciations. Raoul Malard, représentant de la société centrale de magasins à prix uniques, Prisunic, se plaint ainsi au CGQJ de ce que le magasin Nobal, situé au centre de Lens, aux mains de " juifs notoires ", n'ait toujours pas fait l'objet d'une mesure d'aryanisation en 1943 alors qu'il est intéressé par son rachat. Le CGQJ dépêche alors une enquête sur les administrateurs de cette société (ils ont quitté Lens en 1940) ${ }^{24}$. Tel autre propriétaire de Béthune va préférer s'adresser, par l'intermédiaire de son avocat, au bureau des affaires juives de la préfecture le 18 septembre 1942 pour obtenir des renseignements sur un locataire et engager une procédure de recouvrement
de loyers impayés :

"J'ai besoin de savoir pour mettre régulièrement le commissaire gérant en cause le cas échéant, si Henri Lévi est israélite et s'il est nanti d'un commissaire gérant dont vous voudrez bien me donner nom et adresse. Je désirerais aussi que vous me donniez l'adresse à Paris du CGQJ pour renseignements
complémentaires sur Lévi le cas échéant ${ }^{25}$."

Cet exemple illustre avec clarté les mécanismes de l'identification par "réputation ": cette simple demande de renseignement équivaut concrètement à une dénonciation. Dans un premier temps en effet, le bureau de la préfecture répond que cet individu "n'est pas considéré comme israélite ". Mais la missive adressée au CGQJ change la donne et, en janvier 1943, sous la plume du directeur général de l'aryanisation économique, le dénommé Henri L. est devenu «le juif Henri Lévi ». Le CGQJ demande alors que les services locaux procèdent à une enquête dont les résultats sont communiqués le 25 février 1943 par la sous-préfecture : 
"Il était célibataire et n’a jamais fait de déclaration concernant la religion juive. Une de ses vendeuses, la ressortissante polonaise Kwi. Hélène, âgée de 36 ans et demeurant toujours dans un appartement au $2^{\mathrm{e}}$ étage de l'immeuble, a certifié que son ex-patron n'était pas israélite ${ }^{26}$."

Résumons l'affaire : le propriétaire de l'immeuble présume que son locataire est "israélite " alors que la voisine du $2^{\mathrm{e}}$ étage, par ailleurs ex-vendeuse, nie ce fait. Banal conflit de voisinage? L'objectif énoncé par le propriétaire (obtenir le versement des loyers) pourrait le laisser penser. Il n'en est pourtant rien parce que, du point de vue du CGQJ, présomption vaut vérité. La réputation suffit à attester "l'identité » d'un individu, celle-ci étant alors rapidement suivie par un acte d'identification " officielle » : en mai 1943, l'entreprise se voit attribuer un commissaire gérant. Cet exemple illustre le rôle contrasté des voisins dans les processus d'identification, de dénonciation ou encore de " reconnaissance " des " Juifs".

\section{Connaissance, reconnaissance et « réputation »}

Outre la proximité économique ou professionnelle, le voisinage se décline également sur le registre d'un lien interpersonnel de "connaissance ». Fréquenter le même espace géographique, s'il n'est pas en lui-même constitutif de liens entre les individus, implique, dans un cadre local ou micro-local, le fait de "connaître de vue " untel ou untel. Cette connaissance minimale est constitutive d'un savoir sur l'autre dont témoigne la pratique administrative, ancienne dans les services de police, de "l'enquête de voisinage ". La proximité spatiale se décline ici au sein des " micro-sociétés " que délimitent un étage, un immeuble ou encore quelques portions de rues organisées autour de commerces spécifiques et partagés. La notion de " réputation " s'avère centrale dans les enquêtes de "moralité publique", pour reprendre la terminologie déjà utilisée dans les dossiers d'étrangers pendant l'entre-deux-guerres. Dans le contexte de la discrimination propre aux années 1940-1944, le pouvoir de dire la réputation prend un poids particulièrement important. Car on cherche, à tout prix, à connaître, à repérer, à identifier les Juifs. Mais comment reconnaître un juif? Le voisinage connaît-il nécessairement l'appartenance d'untel ou untel à la communauté juive? Rien n'est moins sûr, ce qui n'empêche pourtant pas que les enquêtes de voisinage acquièrent, aux yeux de l'institution policière, le rang de "preuve " de l'appartenance juive. L'identification comme juif repose souvent sur la seule notoriété, dépendante alors du voisinage :

"Le nommé Be. Stephan, né le 25-7-1894 à Bambert, Allemagne, marié, un enfant, est arrivé à Barbazan le 13 novembre 1940, titulaire d'un récépissé de demande de carte d'identité établi à Valenciennes. Il était 
accompagné d'une Française, la nommée CA. Yvonne, née le 6-1-1906 à Douai, Nord. (Cette femme n'était pas juive, mais il est notoire à Barbazan que Bernet l'est, de race et de religion) ${ }^{27}$. "

Pour autant, cette notoriété de voisinage semble ne pas être toujours aussi largement partagée que ce que ses usages policiers laissent supposer. Alors que l'on accuse un dénommé Ma., habitant Harnes, d'avoir contracté avec M. R., commerçant en fruits et légumes désigné comme juif, une transaction commerciale jugée illégale en 1942 (le second a vendu au premier son mulet et sa charrette, désormais inutiles), celui-ci proteste de son ignorance devant le commissaire gérant qui l'interroge : « $\mathrm{M}$. Ma. ignorait, dit-il, que R. qu'il connaissait de vue depuis longtemps était juif et il semble avoir agi de bonne foi ${ }^{28}$. " Protection ou ignorance? Impossible à dire en l'état. Mais au cours de ces années sombres, les voisins ont pu parfois aussi jouer le rôle de protection et d'entraide.

Face à la discrimination, un certain nombre de juifs se tournent en effet vers leurs voisins en quête sinon d'une aide, au moins d'un regard compréhensif pour leur situation. C'est notamment le cas pour ceux qui décident de quitter le Pas-de-Calais, laissant à Lens une partie de leur passé mais souvent, également, de leurs biens. Réfugiés en zone libre, ils envoient alors des lettres à leurs voisins, amis, afin de prendre des nouvelles. Charles S. écrit de Lyon à sa propriétaire et voisine (elle habite le même immeuble à Lens) afin de s'enquérir de sa boutique :

"Lyon le 27 octobre 1941, Chère Madame,

Je vous ai adressé une carte il y a quelques semaines mais aucune réponse ne m'est parvenue jusqu'à ce jours. J'espère quand même que vous allez bien et qu'aucune raison sérieuse ne vous empêche de m'écrire. Je serai bien content d'avoir de vos nouvelles et surtout de savoir comment ça va dans mon magasin. J'ai reçu une carte de $M$. Dubois Pierre 10 rue Bayard qu'il est commissaire gérant de mon magasin. Je vous serai bien obligé de vouloir bien me donner quelques renseignements en ce qui concerne M. Dubois et mes affaires.

En attentant vos nouvelles, je vous prie, chère madame de croire à mes meilleurs sentiments ${ }^{29}$."

Dans ce cas précis, le fait que cette lettre se retrouve dans le dossier d'aryanisation de $S$. n'est pas bon signe : transmise par sa destinataire au commissaire gérant, interceptée par le commissariat ou la préfecture, cette missive n'a jamais eu de réponse. C'est évidemment un des biais importants à prendre en compte dans notre analyse. Les sources produites dans un but discriminatoire offrent un point de vue privilégié pour observer les comportements manifestés dans ce but. Mais il serait erroné de lire l'insertion

27. AN, AJ38/4930.

28. Rapport du commissaire gérant en date du 6 novembre 1942, AN, AJ38/4927.

29. La lettre est reproduite telle qu'elle, AN, AJ38/4975. 
locale des juifs du bassin lensois sous le seul prisme d'un univers hostile. On trouve également, notamment dans les dossiers de restitution des biens le cas échéant, les traces de comportements différents. Les ventes peuvent ainsi être l'occasion d'accords passés entre le commerçant et ses voisins. Michel Fr., négociant en tissus avant la guerre, est installé au 11 bd Basly, à Lens. Les bombardements de 1940 détruisent son local, ses marchandises sont pillées pendant les semaines de désordre qui suivent l'arrivée des troupes allemandes, et il se trouve dans l'impossibilité de reprendre son activité lors de son retour d'exode. Il passe alors un accord avec sa voisine, Mme D., à laquelle il concède le droit de faire les réparations nécessaires pour qu'elle puisse exploiter son propre commerce de rideaux et bonneterie, installé à la même adresse ${ }^{30}$. Puis Fr. quitte Lens pour Paris, non sans passer un nouvel accord écrit avec sa voisine :

"Accord entre les soussignés M. Michel Fr. demeurant actuellement à Paris 10 rue Barbette d'une part et Madame D. Emilia, commerçante à Lens, bd Basly d'autre part,

Il est dit, fait et convenu ce qui suit :

Par suite des événements actuels, Monsieur Michel Fr. ne pouvant rouvrir immédiatement le commerce qu'il exploitait à Lens, 11 bis Bd Basly.

Consent à $\mathrm{M}^{\mathrm{me}}$ Emilia $\mathrm{D}$. dont l'immeuble est détruit pour fait de guerre l'usage des locaux de commerce et d'habitation qu'il occupe à Lens, bd E. B $\mathrm{n}^{\circ} 11$ bis et ce sous les conditions formelles et pour la durée indiquée
ci-après;

Cette concession est faite à titre précaire éminemment révocable, Monsieur Fr. se réservant formellement de reprendre l'usage personnel de ses locaux dès son retour à Lens, mais s'engage toutefois à prévenir Madame D. de son intention au moins quinze jours à l'avance pour lui permettre son déménagement.

De son côté, $\mathrm{M}^{\mathrm{me}} \mathrm{D}$. s'engage et s'oblige formellement à remettre les locaux à $M$. Fr. dès sa première demande sans aucune formalité; Cette clause et condition étant la cause déterminante des présentes conventions et sans laquelle ces dernières n'auraient pas lieu. [suit le descriptif des conditions financières selon lesquelles $\mathrm{M}^{\mathrm{me}} \mathrm{D}$. s'acquitte du loyer auprès du propriétaire des lieux]. Les frais et honoraires des présentes sont à la charge de $\mathrm{M}^{\mathrm{me}} \mathrm{D}$. qui s'y
oblige.

Fait et signé en double exemplaire à Lens, le 4 février $1941^{31}$. "

Au mois d'août de la même année, un commissaire gérant est finalement nommé pour administrer l'entreprise de Fr. qu'il vend, en novembre 1942, à... $\mathrm{M}^{\mathrm{me}} \mathrm{D}$. L'accord passé permet à Michel Fr., qui rentre à Lens en 1945, de récupérer alors son commerce. 30. Lettre du commissaire de police de Lens, en date du 12 mars 1941, au sous-préfet de Béthune,
AN, AJ38/4925, D 4617.

31. Accord sans date, AN AJ38/4925. 
Si les accords sont rarement autant formalisés, d'autres exemples d'entente surgissent parfois au détour d'un dossier. Moïse M., coiffeur à Carvin, demande dès janvier 1941 sa radiation du registre du commerce. C'est une certaine "Mademoiselle P. » (l'une de ses employées?) qui reprend la location du bail et du matériel tout en salariant Moïse ${ }^{32}$. Malgré la suspicion exprimée par le $1^{\text {er }}$ bureau (des affaires juives) de la sous-préfecture dans une note d'août 1942, la nouvelle locataire du bail obtient de l'Oberfeldkommandantur une autorisation d'exercice le 13 juillet 1943. L'arrangement permet ainsi à Moïse, dans un premier temps, de maintenir son activité professionnelle, avant de quitter la localité. Puis, de récupérer son fonds lors de son retour. Le 6 mai 45, il déclare ainsi au chef du service des restitutions : "Je déclare être en possession de mon fonds de commerce dès la Libération. Je considère cette affaire comme classée ${ }^{33}$. "

\section{Laisser ses clés au voisin?}

Régulièrement, les commissaires gérants nommés tardivement ont trouvé porte close devant les entreprises dont ils devaient gérer la spoliation et le transfert en des mains " aryennes ". Bien souvent, en pareil cas, ce furent alors les voisins qui conservaient les clés des locaux. Le commissaire gérant de l'entreprise commerciale d'Alter Ma. s'est rendu le 28 juin 1941 devant la bonneterie-lingerie du 65 bis rue Decrombecque à Lens et a " constaté que le magasin était fermé et l'immeuble inhabité. La clé me fut remise par le voisin immédiat $\mathrm{M}$. L . herboriste à qui elle avait été confiée par M. Ma. L'immeuble que je visitais avec M. L. était vide de meubles et de marchandises. Il subsistait seulement quelques rayonnages et un petit

Les meubles mis en dépôt ont également parfois laissé des traces dans les archives. Ainsi, lorsqu'il apprend que l'immeuble de son beau-frère, parti loin de Lens, doit être réquisitionné pour y installer la permanence du Rassemblement national populaire, Wolf S. s'y rend pour enlever le reste du matériel qui comptait deux comptoirs et quelques rayonnages sans valeur et les place chez M. Lep., gérant des chaussures "Succès » à Lens et voisin ${ }^{35}$.

Ces arrangements locaux sont sans nul doute sous-estimés dès lors que nous échappent l'ensemble de ceux qui "ont réussi » (c'est-à-dire qui n'ont pas été interceptés par l'activité des administrations diligentées par le bureau des " affaires juives "). Chaque fois que l'accord a pu rester clandestin, il nous est également inconnu.

32. Note 789 du commissaire de police de Carvin datée du 8 octobre 1941 qui indique que «M. M. est actuellement le salarié de Mlle P., qui le paye $800 \mathrm{~F}$ par mois " (AN, AJ38/4931).

33. AN AJ38/4931.

34. AN, AJ38/4915, D1675.

35. AN, AJ38/8831. 
Restent quelques traces fugaces qui témoignent de comportements d'entraide. Lorsque les familles partent sans laisser d'adresse, leurs voisins sont sollicités pour connaître leur lieu de destination. Ils peuvent alors se taire. Le sous-préfet précise ainsi à propos d'une enquête sur la disparition de Mosko B., le 9 février 1943 : " Il a quitté furtivement Lens en mars 1941 sans laisser d'adresse et toutes recherches faites à l'époque auprès de ses coreligionnaires en vue de connaître sa nouvelle adresse sont restées sans résultat ${ }^{36}$ ". Voilà la raison pour laquelle il est extrêmement difficile de reconstituer les filières de départ et les étapes et lieux de destination. On sait où les gens sont partis quand ils ont été arrêtés; plus rarement, et en tout cas plus vaguement (souvent sous la seule mention "zone libre») pour ceux qui ne l'ont pas été. En ce sens, seuls les fichiers des camps d'internement et les listes des convois de déportation permettent de reconstituer, et de façon très lacunaire, les chemins de l'exode. Ou encore les témoignages des survivants qui relatent alors, en détail, les réseaux d'entraide, mobilisés à Lens d'abord, pour quitter la place, puis à Paris et dans les lieux de refuge où, la plupart du temps, ils sont attendus, ne serait-ce que par d'anciens voisins, partis plus tôt.

Ce sont précisément ces réseaux locaux d'entraide qui, en septembre 1942, ont fait défaut aux Juifs restés dans le bassin houiller. Au début de cet article, nous avons montré combien l'image d'un petit " ghetto juif " au centre-ville de Lens ne correspondait pas à la réalité. Le point est ici décisif pour comprendre comment l'isolement, adossé au stigmate visant l'immigrant, explique la large indifférence dans laquelle s'est déroulée la rafle du 11 septembre 1942 à Lens. 222 personnes ont été arrêtées ce matin-là (hommes, femmes et enfants) puis, rassemblées, elles ont traversé la ville à pied jusqu’à la gare, encadrées par les soldats allemands ${ }^{37}$, sous le regard de leurs voisins. Les Juifs de Lens n'ont pu compter sur l'appui d'autres Lensois, à la différence notamment de la ville de Lille toute proche, où un nombre non négligeable de personnes ont été prévenues, cachées et ont pu ainsi échapper à la rafle. On sait néanmoins, grâce aux rapports de police, que trois familles (12 personnes) et une enfant disparaissent le 10 septembre $^{38}$, soit la veille de

36. AN, AJ38/4925.

37. S'il semble que les fonctionnaires français n'aient pas participé directement aux arrestations, opérées par les Allemands, ce sont bien eux qui fournissent les listes nominatives et récupèrent, après les arrestations, les denrées périssables et légumes des jardins (voir le "Relevé certifié exact des denrées périssables récupérées chez les juifs arrêtés le 11 septembre, denrées qui ont été remises au Secours national ", commissariat de police de Lens, 18 septembre 1942, ADPC, 1Z497). Puis, le personnel du commissariat établit une nouvelle liste " mise à jour ", rayant les individus arrêtés par les Allemands courant août, comptant et identifiant scrupuleusement ceux qui sont arrêtés le 11 septembre au matin ("Liste des israélites évacués le 11 septembre 1942 ", commissariat, 18 septembre 1942, ADPC, 1Z497), dénombrant, en date du 15 septembre, les 29 personnes qui n'ont pas été " évacuées " ("Israélites existant à Lens au 15 septembre 1942 ", 18 septembre 1942, ADPC, 1Z497), puis ôtant de cette liste les quinze arrestations supplémentaires du 25 (ADPC, 1Z499), pour procéder au recensement final des 13 juifs encore présents à Lens au ler octobre 1942.

38. "Liste des israélites qui ont quitté la localité avant le 11 septembre 1942 sans faire connaître leurs adresses ", 18 septembre 1942, ADPC, 1 Z497. 
la rafle, sans doute prévenues de l'imminence des arrestations. Mais seul un homme parvient à s'échapper le 11 même. Les autres Juifs encore présents en ville sont quant à eux arrêtés, déportés vers Malines, puis Auschwitz.

\section{Conclusion : le retour des voisins}

Tal Bruttmann, dans le dernier chapitre de son livre Au bureau des affaires juives, soulève, brièvement mais de façon très stimulante, la question de la Libération, autrement dit celle du retour à l'ordre républicain : «Et c'est sans doute l'attitude des administrations au sortir de l'Occupation qui permet de mieux révéler la profondeur de l'enracinement de l'antisémitisme ${ }^{39}$. " Faute de pouvoir traiter ici la question de manière approfondie, on se contentera de lancer quelques pistes pour rappeler les immenses difficultés rencontrées par ceux des Juifs qui reviennent (parents ou enfants) dans l'arrondissement de Béthune à partir de l'automne 1944. Que trouvent-ils en revenant chez eux? Certains de leurs anciens voisins, guère prêts à restituer le bien " acquis " lors de la spoliation ou à quitter les lieux. Pour s'en convaincre, il suffit de lire la lettre qu'adresse au sous-préfet le nouveau commissaire à la sécurité publique nommé à Lens à la Libération :

"J'ai l'honneur de vous exposer la situation particulière de la ville de Lens en ce qui concerne les israélites. Il existait ici avant la guerre une colonie de juifs qui occupaient dans le commerce local une situation importante. Certains d'entr'eux ont fait l'acquisition d'immeubles, les autres ont contracté des baux à long termes. Les commerçants français qui les concurrençaient n'ayant pu les écarter avant la guerre ont exploité largement les mesures prises à leur encontre pendant l'occupation et entravent leur réinstallation dans toute la mesure du possible. Les juifs qui ont échappé aux arrestations et reviennent dans la localité ne peuvent récupérer leur mobilier qui a été confisqué et vendu par les domaines ou volé par les allemands ou dispersé. Leurs habitations qui ont été ou détruites ou réquisitionnées soit pour les sinistres, soit pour les services publics. Les fonds de commerce existants encore ont été cédés à d'autres commerçants. Journellement la Police est appelée à intervenir pour apaiser les conflits et éviter les rixes résultant de cette situation que j'ai cru devoir vous signaler, étant donné l'importance de la colonie juive installée à Lens avant la guerre (351 personnes ${ }^{40}$ ) et l'absence de toute instruction officielle s'y rapportant ${ }^{41}$."

Les dossiers de restitution des biens permettent d'éclairer dans le détail la scène décrite par le commissaire de Lens : certains réfugiés vont jusqu’à racheter le bien dont ils ont été spoliés par les mesures d'aryanisation (ainsi

39. Tal Bruttmann, $A$ u bureau..., op. cit., p. 192.

40. Il serait évidemment très intéressant de savoir d'où le commissaire tire ce dénombrement : fichier établi par son prédécesseur (bien que ce chiffre ne corresponde à aucune des listes dont nous dispo-

41. Lettre du 7 décembre 1944 à M. le sous-préfet, ADPC, 12499. 
des héritiers S., dès mai $\left.1946^{42}\right)$. Mais d'autres racontent aussi l'aide que leur apportent d'anciennes connaissances, comme A. J. : " J'ai eu recours, pour me loger, à de braves gens qui ont bien voulu mettre provisoirement à ma disposition, une chambre pour ma femme et pour moi-même. Nous n'avons plus ni logement, ni literie, ni linge, ni ustensiles de ménage, ni atelier de travail, ni marchandises. Nous sommes sur le point d'avoir épuisé toutes nos économies ${ }^{43}$. " On le voit, l'indifférence menace, la désignation condamne, le partage d'informations sauve, parfois. Mais là encore, nous avons tenté de le démontrer, l'affaire ne peut être réduite à l'analyse de la qualité " humaine " du voisinage. C'est à la lumière des inscriptions locales des liens qu'il faut la saisir : autrement dit, il ne s'agit pas de savoir si ce sont de "bons " ou de " mauvais » voisins, mais qui ils sont pour la personne considérée : un propriétaire ou un locataire, un concurrent, un fournisseur ou un client, un parent, un allié ou, à l'inverse, un rival.

42. AN, AJ38/4975.

43. Signé A. J., " marchand tailleur Précédemment à Lens, 69 Bd Basly, actuellement à Lens 41 rue de L'Abattoir ", Lens, 24 février 1945, AN, AJ38/4927. 